\title{
TCAD oriented simulation of single-electron transistors at device level
}

\author{
A. Scholze, A. Schenk, and W. Fichtner \\ Integrated Systems Laboratory, ETH-Zürich \\ ETH Zentrum, CH-8092 Zürich, Switzerland
}

\begin{abstract}
In this paper we present a simulation approach for electron transport in singleelectron devices based on a weak-coupling formulation for the linear-response transconductance of a quantum dot/reservoir system. A simulation tool devised for the simulation of single-electron transistors has been developed. It provides the equilibrium solution of the nonlinear Pojsson equation for the classical charges in the bulk and the self-consistent solution of the 3D-SchrödingerPoisson equation for the quantum dot. The finite temperature groundstate of the few-electron ensemble in the dot is extracted by evaluation of the Gibbs distribution. The program is coupled to a 3D modeling tool for flexible geometry specification.
\end{abstract}

\section{Introduction}

Single-clectron transistors (SET) are an emerging class of devices for potential memory and logic applications. Two of the key concepts in the operation of single-electron devices are that of the Coulomb blockade and of single-electron tunneling. The sim-

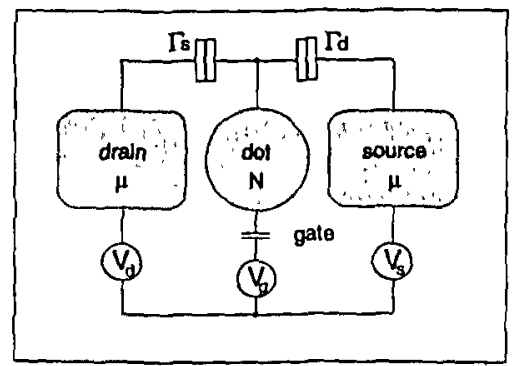

Figure 1: Schematic view of the circuit representing a two-terminal single electron transistor. The dot is coupled to the reservoirs via tunneling junctions while the coupling to the gate is capacitive.

plest model for a single-electron tunneling structure consists of a reservoir weakly coupled to a quantum dot. A basic transistor structure (Figure 1) consists of two 
independent reservoirs which are coupled to the dot via tunneling junctions. The potential in the dot depends on one or more capacitively coupled gate electrodes. Most present effort towards simulation of single-electron effects in nanostructures is circuit oriented and based on semi-classical models for the self-capacitance of metal islands (e.g. [1]). In semiconductor SETs, however, the discrete nature of the electron states makes a self-consistent analysis of a electron ensemble in a strong $0 D$ confinement necessary. Furthermore, assumptions regarding the appropriate carrier statistics have to be shifted from purely grand-canonical to canonical ensemble theory, appropriate for the description of systems with a few electrons under the influence of external reservoirs.

\section{Numerical methods}

In this paper, a simulation tool devised for the simulation of SETs at the solid-state device level is presented. The screening of the charges in the bulk is described by means of a classical Thomas-Fermi model for electrons and holes in equilibrium. A

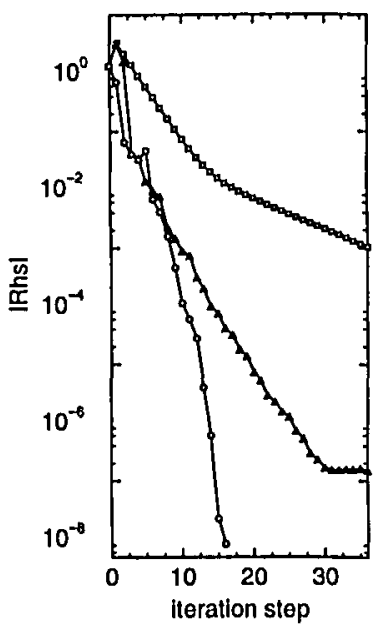

Figure 2: Performance of the quasi-Newton algorithm (o) versus a simple mixing method $(\square)$ and Anderson mean-value mixing $(\Delta)$.

standard Newton-Raphson method is employed to solve for the electrostatic potential $\phi(\rho)$, treating the dot charge classically. In a second step, the dot charge is replaced by charges arising from discrete electron occupation numbers, and a 3D-SchrödingerPoisson equation is solved for each occupation. The nonlinear problem is formulated in terms of a multidimensional root search for a vector function with the values of the potential at discrete mesh points as argument

$$
\mathbf{F}\left(\phi(\rho), v_{\mathrm{xc}}[n]\right)=0 .
$$

$\rho$ is the total carrier density, $n$ the electron density and $F$ the vector function whose root is sought. A local approximation [2] to the exchange-correlation potential $v_{\mathrm{xc}}$ is used to account for quantum effects in the electron-electron interaction. This approach is justified if the number of bulk charges by far exceeds the number of 
charges in the dot, and therefore, changes in the electrostatic potential are significant in the dot region only.

A powerful quasi-Newton method (Broyden method [3]) to minimize the residual of highly nonlinear sets of equations has been implemented. The convergence is not quadratic as with Newton-Raphson methods. However, superlinear convergence is achieved. The method is thus superior in performance to underrelaxation methods. Apart from the convergence rate, the stability of the self-consistent iteration has also been improved. We use a preconditioned bistable conjugate gradient method as the linear solver and a very effective Jacobian-Davidson QR algorithm to solve for the lowest eigenvalues of a sparse matrix.

\section{Physical aspects}

The Gibbs distribution $P_{\mathrm{eq}}\left(\left\{n_{i}\right\}\right)$ of the electron population in a quantum dot in equilibrium with the reservoirs is evaluated in order to find the true many particle ground state of the system of dot and reservoir

$$
P_{\mathrm{eq}}\left(\left\{n_{i}\right\}\right)=\frac{\exp \left[-\left(F\left(\left\{n_{i}\right\}, N\right)-E_{\mathrm{F}} N\right) / k_{\mathrm{B}} T\right]}{\sum_{\left\{n_{\mathrm{i}}\right\}} \exp \left[-\left(F\left(\left\{n_{i}\right\}, N\right)-E_{\mathrm{F}} N\right) / k_{\mathrm{B}} T\right]}
$$

where $F\left(\left\{n_{i}\right\}, N\right)$ denotes the total free energy of the system with $N$ electrons and the occupation configuration $\left\{n_{i}\right\},\left(n_{i}=0,1\right)$ and $E_{\mathrm{F}}$ is the Fermi-energy, respectively. Key parameters for the device operation such as the thermodynamical average of the number of electrons in the quantum dot

$$
\langle N\rangle=\sum_{\left\{n_{1}\right\}} P_{\mathrm{eq}}\left(\left\{n_{i}\right\}\right) N \delta_{N, \sum_{i} n_{1}}
$$

or the differential capacitance

$$
C_{\mathrm{d}}=e^{2} \frac{\partial\langle N\rangle}{\partial \mu} \text {. }
$$

can be extracted. The quantity $\iota=F(N+1)-F(N)$ defines the chemical potential of the dot. Equation (4) in the zero temperature limit corresponds to the equation for the differential capacitance given by Macuchi et al. [4]:

$$
C_{\mathrm{d}}=e^{2}[\mu(N)-\mu(N-1)]^{-1} .
$$

In addition we calculate the linear response transconductance through the confinement region using the solution to the set of detailed balance equations (master equation) derived by Beenakker [5]

$$
G=\frac{e^{2}}{k_{\mathrm{B}} T} \sum_{\left\{n_{\mathrm{l}}\right\}} P_{e q}\left(\left\{n_{\imath}\right\}\right) \sum_{k} \delta_{n_{k}, 0} \frac{\Gamma_{k}^{\mathrm{s}} \Gamma_{k}^{\mathrm{d}}}{\Gamma_{k}^{\mathrm{s}}+\Gamma_{k}^{\mathrm{d}}} f\left(F\left(\left\{n_{\imath}+k\right\}, N+1\right)-F\left(\left\{n_{\mathrm{\imath}}\right\}, N\right)-E_{\mathrm{F}}\right) .
$$

where $\Gamma^{\mathrm{d}}$ and $\Gamma^{\mathrm{s}}$ are the tunneling rates for the drain- and source-side barrier, respectively. They are estimated using Fermi's golden rule. Each data point of the transconductance characteristics requires the evaluation of a set of total free energies $F\left(\left\{n_{1}\right\}, N\right)$, which result from the self-consistent evaluation of the screening. At low temperatures it is possible to restrict the summation in the Gibbs distribution to those configurations $n_{2}$ whose total free energy is close to the minimum $F_{\min }$. In the zero 
temperature limit of the differential capacitance (5), the condition for the appearance of a conductance peak can be reduced to the degeneracy of two values of $F$. The position of the conductance peaks is then equivalent to the difference of the total free energies at $N$ and $N-1$. Peaks are sufficiently described by delta functions (Figure $3)$. However, with increasing temperature a wider range of occupation configurations becomes relevant and the numerical effort increases likewise. Thus, numerical effectiveness and stability of the self-consistent iteration is a key requirement.

\section{Conclusions and outlook}

We have connected the simulator to a tensor-product mesh editor that allows a high degree of freedom with respect to geometry modeling and boundary description. In

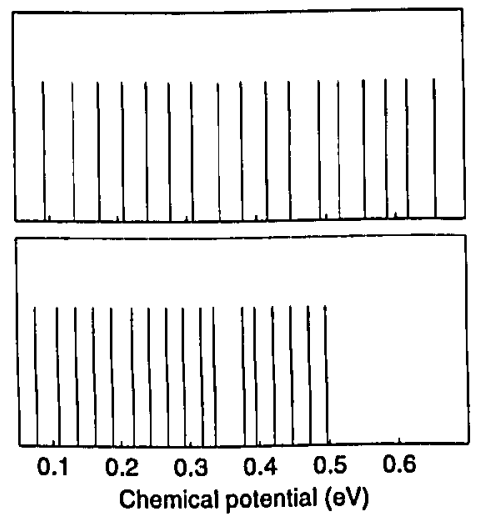

Figure 3: Position of conductance peaks for a test structure at $\mathrm{T}=0 \mathrm{~K}$. The dot diameter is increased from $10 \mathrm{~nm}$ at the upper panel to $25 \mathrm{~nm}$ at the lower panel.

general it is planned to incorporate the simulator in an existing TCAD environment of ISE AG Zürich. An interface to a conventional device simulator could extend the class of devices that can be tackled considerably.

\section{References}

[1] C. Wasshuber, H. Kosina and S. Selberherr, "A Single Electron Device and Circuit Simulator with a New Algorithm to Incorporate Co-tunneling", Proc. of SISPAD'96, pp. 135-136, 1996.

[2] J. P. Perdew and A. Zunger, "Self-interaction correction to density-functional approximations for many-electron systems", Phys. Rev. B, vol. 23, pp. 5048-5079, 1981.

[3] D. D. Johnson, "Modified Broyden's method for accelerating convergence in selfconsistent calculations", Phys. Rev. B, vol. 38, pp. 12 807-12 813, 1988.

[4] M. Macucci, K. Hess and G. J. Iafrate, "Electronic energy spectrum and the concept of capacitance in quantum dots", Phys. Rev. B, vol. 48, pp. 17 354-17 363, 1993.

[5] C. W. J. Becnakker, "Theory of Coulomb-blockade oscillations in the conductance of a quantum dot", Phys. Rev. B, vol. 44, pp. 1646-1656, 1991. 